ARTICLE

\title{
Dynamic fingerprint of fractionalized excitations in single-crystalline $\mathrm{Cu}_{3} \mathrm{Zn}(\mathrm{OH})_{6} \mathrm{FBr}$
}

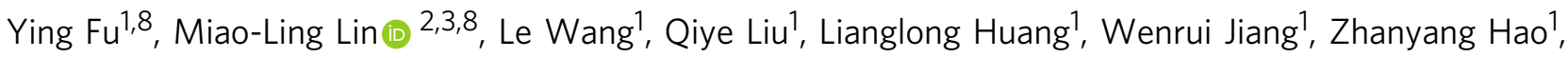
Cai Liu (1) ${ }^{1}$, Hu Zhang ${ }^{4}$, Xingqiang Shi ${ }^{4}$, Jun Zhang (1) ${ }^{2,3,5}$, Junfeng Dai ${ }^{1}$, Dapeng Yu ${ }^{1}$, Fei Ye ${ }^{1,6}$, Patrick A. Lee ${ }^{7}$, Ping-Heng Tan (i) ${ }^{2,3,5 凶}$ \& Jia-Wei Mei (iD ${ }^{1,6 \times}$

Beyond the absence of long-range magnetic orders, the most prominent feature of the elusive quantum spin liquid (QSL) state is the existence of fractionalized spin excitations, i.e., spinons. When the system orders, the spin-wave excitation appears as the bound state of the spinon-antispinon pair. Although scarcely reported, a direct comparison between similar compounds illustrates the evolution from spinon to magnon. Here, we perform the Raman scattering on single crystals of two quantum kagome antiferromagnets, of which one is the kagome QSL candidate $\mathrm{Cu}_{3} \mathrm{Zn}(\mathrm{OH})_{6} \mathrm{FBr}$, and another is an antiferromagnetically ordered compound $\mathrm{EuCu}_{3}(\mathrm{OH})_{6} \mathrm{Cl}_{3}$. In $\mathrm{Cu}_{3} \mathrm{Zn}(\mathrm{OH})_{6} \mathrm{FBr}$, we identify a unique one spinon-antispinon pair component in the $E_{2 g}$ magnetic Raman continuum, providing strong evidence for deconfined spinon excitations. In contrast, a sharp magnon peak emerges from the one-pair spinon continuum in the $E_{\mathrm{g}}$ magnetic Raman response once $\mathrm{EuCu}_{3}(\mathrm{OH})_{6} \mathrm{Cl}_{3}$ undergoes the antiferromagnetic order transition. From the comparative Raman studies, we can regard the magnon mode as the spinon-antispinon bound state, and the spinon confinement drives the magnetic ordering.

\footnotetext{
${ }^{1}$ Shenzhen Institute for Quantum Science and Engineering, and Department of Physics, Southern University of Science and Technology, Shenzhen, China. ${ }^{2}$ State Key Laboratory of Superlattices and Microstructures, Institute of Semiconductors, Chinese Academy of Sciences, Beijing, China. ${ }^{3}$ Center of Materials Science and Optoelectronics Engineering \& CAS Center of Excellence in Topological Quantum Computation, University of Chinese Academy of Sciences, Beijing, China. ${ }^{4}$ College of Physics Science and Technology, Hebei University, Baoding, China. ${ }^{5}$ Beijing Academy of Quantum Information Science, Beijing, China. ${ }^{6}$ Shenzhen Key Laboratory of Advanced Quantum Functional Materials and Devices, Southern University of Science and Technology, Shenzhen, China. ${ }^{7}$ Department of Physics, Massachusetts Institute of Technology, Cambridge, MA, USA. ${ }^{8}$ These authors contributed equally: Ying Fu,

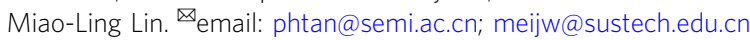


uantum spin liquid (QSL) represents a new class of condensed matter states characterized by the long-range many-body entanglement of topological orders ${ }^{1-9}$. The lattice of the spin-1/2 kagome network is a long-sought platform for antiferromagnetically interacting spins to host a QSL ground state $^{10-16}$. However, a structurally ideal realization of the kagome lattice in experiments is rare. Herbersmithite $\left[\mathrm{ZnCu}_{3}(\mathrm{OH})_{6} \mathrm{Cl}_{2}\right]$ is the first promising kagome QSL candidate ${ }^{3,16-23}$, in which no long-range magnetic order was detected down to low temperature ${ }^{17,18}$, and inelastic neutron scattering revealed a magnetic continuum, as a hallmark of fractionalized spin excitations ${ }^{20,22}$. Up to date, most, if not all, experimental information on the nature of kagome QSL relies on a single compound of Herbertsmithite. Considering the fact that a lattice distortion has recently been confirmed in Herbersmithite ${ }^{24,25}$, which stimulates investigations on the subtle magneto-elastic effect in the kagome materials ${ }^{26,27}$, an alternative realization of the QSL compound with the ideal kagome lattice is still in urgent need. $\mathrm{Zn}$-Barlowite $\left[\mathrm{Cu}_{3} \mathrm{Zn}(\mathrm{OH})_{6} \mathrm{FBr}\right]$ is another candidate for a kagome QSL ground state ${ }^{28-38}$ with no lattice distortion being reported yet. Measurements on the powder samples didn't detect the long-range magnetic order down to temperatures of $0.02 \mathrm{~K}$, four orders of magnitude lower than the Curie-Weiss temperature ${ }^{30,32}$. Besides the lack of magnetic order, the fractionalized spin excitations, i.e., spinons, is essential evidence for the long-range entanglement pattern in QSL. However, spectroscopic evidence for the deconfined spinon excitations in $\mathrm{Zn}$ Barlowite is still lacking, in part due to unavailable single-crystal samples.

Raman scattering is sensitive to the local symmetries depending on the light polarization ${ }^{39,40}$, and also capable of detecting magnetic excitations ranging from the spin-wave magnon excitation to deconfined spionons ${ }^{41-50}$. Raman scattering has previously been reported for Herbertsmithite and revealed the multiple spinon scattering process ${ }^{19}$. In recent years, the atacamite family $\mathrm{ReCu}_{3}(\mathrm{OH})_{6} \mathrm{Cl}_{3}(\mathrm{Re}=\mathrm{Y}, \mathrm{Eu}, \mathrm{Sm}$, and $\mathrm{Nd})$ with the perfect kagome lattice has been synthesized and a chiral $120^{\circ}$ antiferromagnetic (AFM) order with the wave vector $\mathbf{q}=0$ is identified in the ground state $\mathrm{e}^{51-55}$. The kagome spin systems can be described by the kagome Heisenberg model with the Dzyaloshinski-Moriya (DM) interaction

$$
H=J \sum_{\langle i j\rangle}\left(\mathbf{S}_{i} \cdot \mathbf{S}_{j}\right)+D \hat{z} \cdot \sum_{\langle i j\rangle} \mathbf{S}_{i} \times \mathbf{S}_{j},
$$

where summation runs over nearest-neighbor bonds $\langle i j\rangle$, and $J$ and $D$ are the nearest-neighbor exchange and the DM interaction constants, respectively, for the spins $S_{i, j}$ on the $i$ - and $j$-th sites. We ignore the in-plane DM interactions regarding to the previous electron paramagnetic resonance measurements in the related kagome systems ${ }^{55,56}$. A DM interaction larger than the critical value of $(D / J)_{c} \sim 0.08$ induces a chiral $120^{\circ} \mathrm{AFM}$ order from the QSL state ${ }^{57-59}$. By the first-principle calculations (Supplementary Note 1), $\mathrm{Zn}$-Barlowite and $\mathrm{EuCu}_{3}(\mathrm{OH})_{6} \mathrm{Cl}_{3}$ have $\mathrm{D} / J$ values of 0.05 and 0.3 , resulting in QSL and AFM ground states, respectively, consistent with the experimental identification of the ground states ${ }^{30,54}$. While the elementary spin excitation of the kagome QSL is the deconfined spinon, the low energy excitation in the kagome AFM ordered states is the magnon. A direct comparison by the magnetic Raman scattering can reveal the evolution from deconfined spinons in $\mathrm{Zn}$-Barlowite to magnons in $\mathrm{EuCu}_{3}(\mathrm{OH})_{6} \mathrm{Cl}_{3}$, but has not been performed yet.

In this work, we exclude the kagome lattice distortion by angleresolved polarized Raman (ARPR) scattering and secondharmonic-generation ( $\mathrm{SHG}$ ), and reveal the spin dynamics of spinon excitations on the single-crystalline $\mathrm{Cu}_{3.18} \mathrm{Zn}_{0.82}(\mathrm{OH})_{6} \mathrm{FBr}$. We observe a remarkable $E_{2 \mathrm{~g}}$ magnetic Raman continuum, which can be decomposed into one spinon-antispinon pair (one-pair (1P)) and two spinon-antispinon pair (two-pair (2P)) components of spinon excitations, in line with theoretical studies of the kagome QSL ${ }^{60}$. The one-pair continuum is unique, serving as the fingerprint of spinons. In a control experiment, beside the twomagnon (2M) magnetic Raman continuum, we probe a sharp one-magnon $(1 \mathrm{M})$ Raman peak in $\mathrm{EuCu}_{3}(\mathrm{OH})_{6} \mathrm{Cl}_{3}$ below the AFM transition temperature. The magnon peak emerges from the $1 \mathrm{P}$ continuum in the magnetic Raman scattering, can be regarded as the bound state of the spinon-antispinon excitations. As schematically summarized in Fig. 1, our comparative Raman study demonstrates the spinon deconfinement and confinement in the kagome QSL compound and ordered antiferromagnet, respectively. The AFM order transition can be thought to be driven by the spinon confinement.

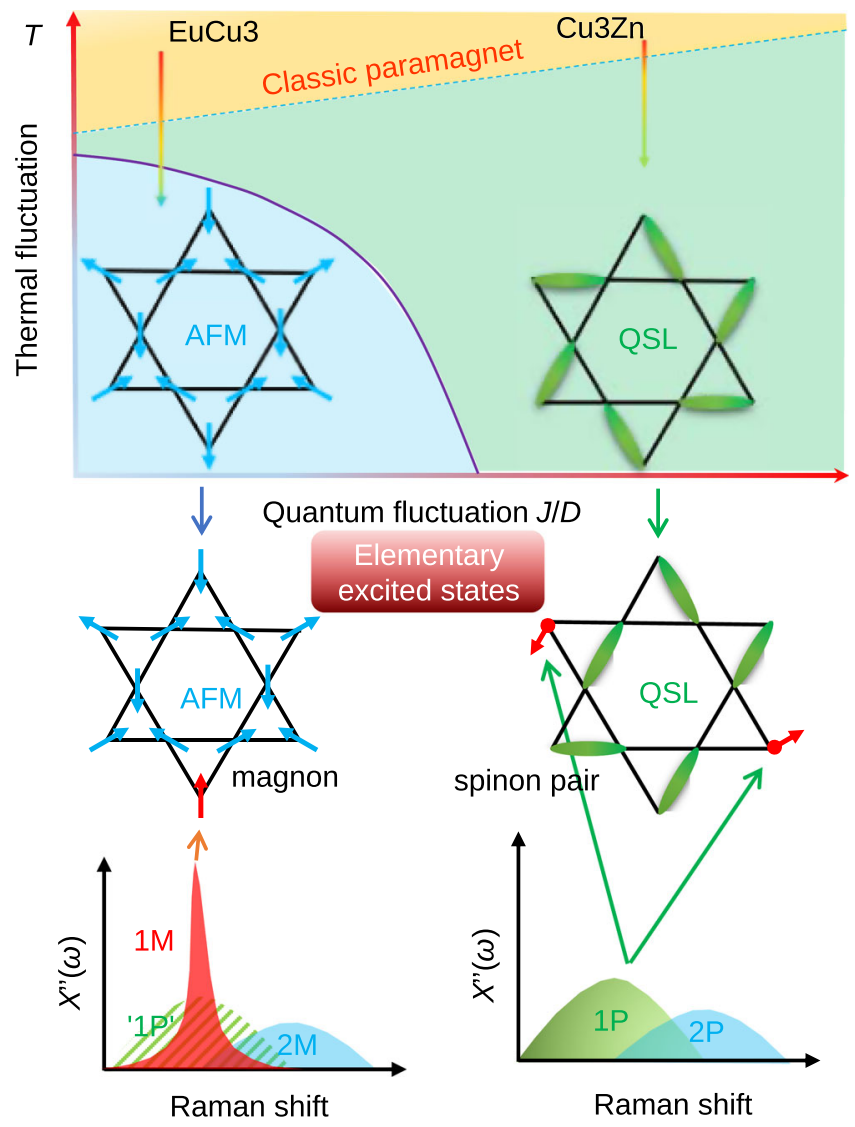

Fig. 1 Schematical comparative Raman responses for the AFM and QSL states. With a large DM interaction $D$, the kagome antiferromagnet develops a chiral $120^{\circ}$ AFM ground state. Increasing $J / D$, the fluctuation of the kagome system increases, driving the system into the QSL state. By increasing the temperature, the thermal fluctuation melts the magnetic order and turns the system into the classic paramagnetic state at high temperatures. $\mathrm{Cu}_{3} \mathrm{Zn}$ and $\mathrm{EuCu}_{3}$ have the QSL and AFM ground states, and allow spinon and magnon excitations, respectively. Magnetic Raman scattering measures different elementary excited states in the two different ground states. Here $1 \mathrm{P}$ and $2 \mathrm{P}$ denote the one-pair and two-pair spinon excitations, respectively. $1 \mathrm{M}$ and $2 \mathrm{M}$ in AFM ordered state denote the oneand two-magnon excitations, respectively. The $1 \mathrm{M}$ Raman peak in AFM measures the magnon while the 1P Raman continuum in QSL probes the spinon excitations. The shadow background of the $1 \mathrm{M}$ peak, marked as ' $1 \mathrm{P}$ ', denotes the continuum above $T_{\mathrm{N}}$ in $\mathrm{EuCu}_{3}$, mimicking the $1 \mathrm{P}$ continuum in the QSL state. 


\section{Results}

We grown single crystals of Barlowite $\mathrm{Cu}_{4}(\mathrm{OH})_{6} \mathrm{FBr}, \mathrm{Zn}$ Barlowite $\mathrm{Cu}_{3.18} \mathrm{Zn}_{0.82}(\mathrm{OH})_{6} \mathrm{FBr}$, and $\mathrm{EuCu}_{3}(\mathrm{OH})_{6} \mathrm{Cl}_{3}$ (we use the short-hand notation $\mathrm{Cu}_{4}, \mathrm{Cu}_{3} \mathrm{Zn}$, and $\mathrm{EuCu}_{3}$, respectively) with high quality ("Methods" and Supplementary Note 2). The interlayer $\mathrm{Cu}^{2+}$ concentration $(18 \%)$ is comparable to that (15\%) in Herbertsmithite ${ }^{61}$. We estimate the superexchange strength for the kagome spins in $\mathrm{Cu}_{3} \mathrm{Zn}$ as $J \simeq 13 \mathrm{meV}$ by the Curie-Weiss temperature $\Theta_{\mathrm{CW}}=-220 \mathrm{~K}$ (Supplementary Note 2) ${ }^{62}$. The superexchange interaction for $\mathrm{EuCu}_{3}$ is about $J \simeq 7 \mathrm{meV}^{53-55}$. Note the electronic ground state of $\mathrm{Eu}^{3+}$ in $\mathrm{EuCu}_{3}$ is the nonmagnetic ${ }^{7} F_{0}$ configuration.

Figure $2 \mathrm{a}$ presents the temperature evolution of Raman spectra in $\mathrm{Cu}_{3} \mathrm{Zn}$ with sharp phonon modes superimposing on the magnetic continuum background. With the help of firstprinciples calculations, we assign the symmetry representations for phonon modes in Supplementary Note 3. No structural phase transition is observed in $\mathrm{Cu}_{3} \mathrm{Zn}$ down to $4 \mathrm{~K}$. We tracked the Raman spectral evolution of the crystal structures from $\mathrm{Cu}_{4}$ to $\mathrm{Cu}_{3} \mathrm{Zn}$ (Supplementary Note 4 ). $\mathrm{Cu}_{3} \mathrm{Zn}$ has no Raman-active mode related to the kagome $\mathrm{Cu}^{2+}$ vibrations, indicating the kagome layer remains intact. $\mathrm{Cu}_{4}$ has distorted kagome layers at $200 \mathrm{~K}$, signaled by an extra phonon mode at $62 \mathrm{~cm}^{-1}$ corresponding to the kagome $\mathrm{Cu}^{2+}$ vibration. The previous $\mathrm{SHG}$ study revealed the parity symmetry in Barlowite $2\left[\mathrm{Cu}_{4}(\mathrm{OH})_{6} \mathrm{FBr}\right]$ and Zn-Barlowite $\left[\mathrm{Cu}_{3.66} \mathrm{Zn}_{0.33}(\mathrm{OH})_{6} \mathrm{FBr}\right]^{25}$. We confirmed the inversion symmetry by SHG in our single crystals of $\mathrm{Cu}_{3} \mathrm{Zn}$ (Supplementary Note 6).

Figures $2 \mathrm{~b}-\mathrm{d}$ are the ARPR responses of $\mathrm{Cu}_{3} \mathrm{Zn}$ in three different polarization configurations ("Methods" and Supplementary Note 5). In the XX (XY) configuration, the incoming and outgoing light polarizations are parallel (perpendicular) and rotated simultaneously. In the X-only configuration, the outgoing polarization is fixed and only incoming light is rotated. Theoretically, the Raman cross section of a Kagome QSL ground state does not depend on the polarization of the incoming or outgoing light ${ }^{39}$ and keeps invariant against rotating light polarization in the $\mathrm{XX}, \mathrm{XY}$, and $\mathrm{X}$ only configurations. Figure $2 \mathrm{~b}$ is the ARPR response for the magnetic continua at low frequency with the integrated Raman susceptibility $\chi^{\prime}=\frac{2}{\pi} \int_{10 \mathrm{~cm}^{-1}}^{60} \frac{\chi^{\prime \prime}(\omega)}{\omega} d \omega$, where the susceptibility is related to the Raman intensity $I(\omega)=(1+n(\omega)) \chi^{\prime \prime}(\omega)$ with the bosonic temperature factor $n(\omega)$. Figure $2 \mathrm{c}$ and $\mathrm{d}$ are the corresponding results of the $\mathrm{Br}^{-} E_{2 \mathrm{~g}}$ phonon, and $\mathrm{O}^{2-} A_{1 \mathrm{~g}}$ phonon modes, respectively. For threefold rotation symmetry, the $A_{1 \mathrm{~g}}$ mode response follows the $\cos ^{2}(\theta)$ function of the rotation angle $\theta$ in X-only configuration, keeps constant in XX polarization, and vanishes in $\mathrm{XY}$ configuration; the $E_{2 \mathrm{~g}}$ mode is isotropic in all the three configurations. The magnetic continuum contains both $A_{1 \mathrm{~g}}$ and $E_{2 g}$ channels at high temperature $(290 \mathrm{~K})$, and only the $E_{2 \mathrm{~g}}$ channel at low temperature $(4 \mathrm{~K})$. The experimental ARPR responses agree well with the theoretical dash-dotted curves, confirming the threefold rotational symmetry in the magnetic excitations (Fig. 2b) and lattice vibrations (Figs. 2c, d). We notice that in Herbertsmithite, although it was not discussed, the lattice distortion was evident by the anisotropic ARPR responses ${ }^{19}$ and may account for the difference from our results.

Having established the structurally ideal realization of the kagome lattice by SHG and ARPR scattering, and the absence of the thermodynamic anomaly, we now present our spectroscopic results of spin dynamics in $\mathrm{Cu}_{3} \mathrm{Zn}$ with subtracting phonon contributions. Figure $3 \mathrm{a}-\mathrm{c}$ are the magnetic continuum of $\mathrm{Cu}_{3} \mathrm{Zn}$ in the $A_{1 \mathrm{~g}}$ channel, which is activated only at high temperatures, and disappears at low temperatures. The integrated Raman susceptibility in Fig. $3 \mathrm{~b}$ fits the thermally activated function, $\chi^{\prime}(T) \propto$ $\mathrm{e}^{-\omega^{*} / T}$ with $\omega^{*}=53 \mathrm{~cm}^{-1}$. The result suggests the $A_{1 \mathrm{~g}}$ continuum measures the thermal fluctuation of the interacting kagome a
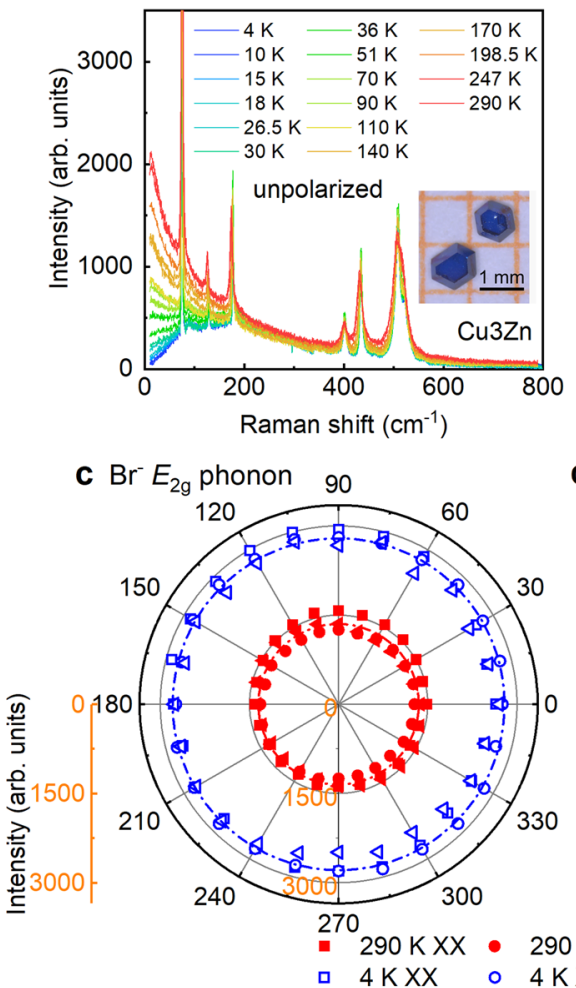

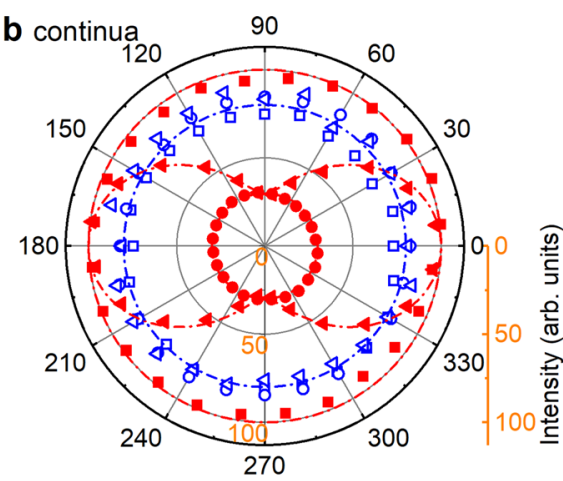

d $\mathrm{O}^{2-} A_{\mathrm{g}}$ phonon

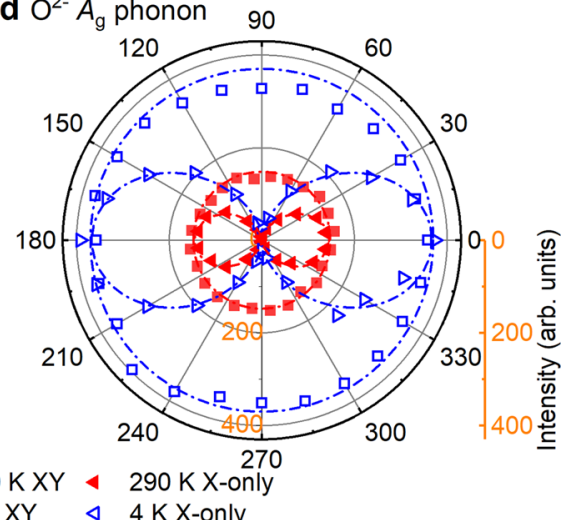

Fig. 2 Temperature dependent and ARPR spectra in $\mathbf{C u}_{\mathbf{3}} \mathbf{Z n}$. a Temperature evolution of unpolarized Raman spectra in $\mathrm{Cu}_{3} \mathbf{Z n}$. The inset is the photo of single crystals. ARPR intensity for low-energy continua (b), the $\mathrm{Br}^{-} E_{2 \mathrm{~g}}$ phonon $\left(75 \mathrm{~cm}^{-1}\right)(\mathbf{c})$, and the $\mathrm{O}^{2-} A_{1 \mathrm{~g}}$ phonon $\left(429 \mathrm{~cm}^{-1}\right)(\mathbf{d})$. The dash-dotted lines are the corresponding theoretical curves based on the $C_{3}$ rotation symmetry. 

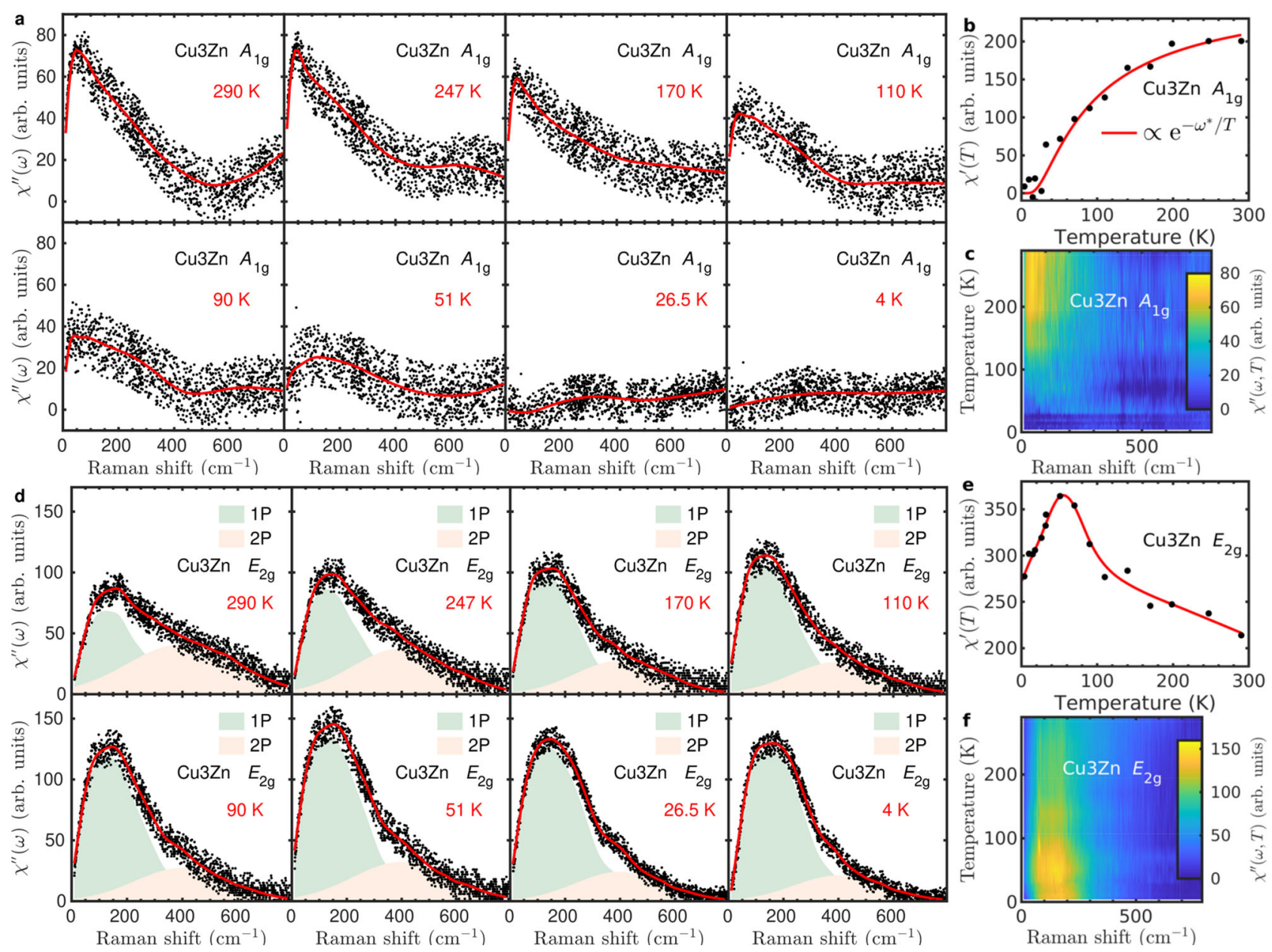

Fig. 3 Temperature dependent magnetic Raman continua in $\mathbf{C u}_{\mathbf{3}} \mathbf{Z n}$. a The $A_{\mathrm{g}}$ Raman susceptibility $\chi_{A_{1 \mathrm{~g}}}^{\prime \prime}=\chi_{\mathrm{Xx}}^{\prime \prime}-\chi_{\mathrm{XY}}^{\prime \prime}$. The solid lines are guides to the eye. b Temperature dependence of the $A_{1 g}$ static Raman susceptibility $\chi_{A_{1 g}}^{\prime}(T)=\frac{2}{\pi} \int_{10 \mathrm{~cm}^{-1}}^{400 \mathrm{~cm}^{-1} \chi_{A_{\mathrm{g}}}^{\prime \prime}(\omega)} \frac{\omega}{\omega} d \omega$. The solid line is a thermally activated function. c Color map of $\chi_{A_{1 g}}^{\prime \prime}(\omega, T)$. d The $E_{2 g}$ Raman response function $\chi_{E_{2 g}}^{\prime \prime}=\chi_{X Y}^{\prime}$. The solid lines are guides to the eye. The light green and pink shadow marked as "1P" and " $2 \mathrm{P}^{\prime \prime}$ represent the one-pair and two-pair components of Raman continuum. e Temperature dependence of the $E_{2 g}$ static Raman susceptibility

$\chi_{E_{2 g}}^{\prime}(T)=\frac{2}{\pi} \int_{10 \mathrm{~cm}^{-1}}^{780 \mathrm{~cm}^{-1}} \frac{\chi_{E_{2 g}}^{\prime \prime}(\omega)}{\omega} d \omega$. The solid line is a guide to the eye. $\mathbf{f}$ Color map of $\chi_{E_{2 g}}^{\prime \prime}(\omega, T)$.

spins ${ }^{41,63,64}$. Different from the $A_{1 \mathrm{~g}}$ channel, the pronounced $E_{2 \mathrm{~g}}$ magnetic Raman continuum persists down to $4 \mathrm{~K}$ (Fig. $3 \mathrm{~d}-\mathrm{f}$ ), indicating the intrinsic quantum fluctuation of the kagome spins. The substantial low energy component has a non-monotonic temperature dependence. It increases with the temperature decreasing from $290 \mathrm{~K}$ to $50 \mathrm{~K}$, but decreases with further temperature reducing as shown in Fig. $3 \mathrm{~d}-\mathrm{f}$. The $E_{2 \mathrm{~g}}$ magnetic Raman susceptibility $\chi^{\prime \prime}(\omega, T)$ distributes the main spectral weight among the frequency region less than $400 \mathrm{~cm}^{-1}$, and reaches the maximum at around $150 \mathrm{~cm}^{-1}$ and $50 \mathrm{~K}$, as shown in Fig. $3 \mathrm{f}$.

The low-energy $E_{2 \mathrm{~g}}$ Raman continuum is crucial as it has an origin of the spinon excitation in the kagome QSL from the theoretical perspective ${ }^{60}$. In the XY configuration for the $E_{2 \mathrm{~g}}$ channel, the Raman tensor on the kagome lattice is written in terms of spin-pair operators $39,60,65,66$

$$
\tau_{R} \propto \sum_{R} \mathbf{S}_{R 3} \cdot\left(\mathbf{S}_{R 1}+\mathbf{S}_{R+\mathbf{a}_{2} 1}-\mathbf{S}_{R 2}-\mathbf{S}_{R-\mathbf{a}_{1}+\mathbf{a}_{2} 2}\right),
$$

where $\boldsymbol{S}_{R 1,2,3}$ are spin operators on three sites of the $R$-th kagome unit cell and $\mathbf{a}_{1,2}$ are the lattice vectors. The spin operator has the spinon $f_{i \sigma}$ representation $S_{i}^{\alpha}=\sum_{\sigma \sigma^{\prime}} f_{i \sigma}^{\dagger} \tau_{\sigma \sigma^{\prime}}^{\alpha} f_{i \sigma^{\prime}} / 2$ where $\tau^{\alpha}$ is the $\alpha$ th Pauli matrix. The spin-pair is $\mathbf{S}_{i} \cdot \mathbf{S}_{j}=-\frac{1}{2} \hat{\chi}_{i j}^{\dagger} \hat{\chi}_{i j}$ with $\hat{\chi}_{i j}=\sum_{\sigma} f_{i \sigma}^{\dagger} f_{j \sigma}$. In the mean field theory, the spinon hopping amplitude $\chi=\left\langle\hat{\chi}_{i j}\right\rangle$ is non-zero. So we have $1 \mathrm{P}$ and $2 \mathrm{P}$ components in the Raman tensor 60

$$
\begin{gathered}
\tau_{R}^{1 \mathrm{P}} \propto \chi \sum_{R}\left(\hat{\chi}_{R 3, R 1}+\text { h.c. }\right)+\cdots, \\
\tau_{R}^{2 \mathrm{P}} \propto \sum_{R} \hat{\chi}_{R 3, R 1}^{\dagger} \hat{\chi}_{R 3, R 1}+\cdots,
\end{gathered}
$$

where $\cdots$ denotes omitted terms in Eq. (2) for the notation simplicity. While the $2 \mathrm{P}$ component is analogous to the $2 \mathrm{M}$ scattering, the $1 \mathrm{P}$ contribution is a unique prediction for spinon excitations in the kagome QSL. In Fig. 3d, we schematically decompose the $E_{2 \mathrm{~g}}$ Raman continuum into $1 \mathrm{P}$ and $2 \mathrm{P}$ components of spinon-antispinon excitations. The $1 \mathrm{P}$ component has the maximum at $150 \mathrm{~cm}^{-1}(1.4 J)$, and extends up to $400 \mathrm{~cm}^{-1}$ $(3.8 J)$ at low temperatures. The $2 \mathrm{P}$ component has the maximum at $400 \mathrm{~cm}^{-1}(3.8 J)$ and the cut-off around $750 \mathrm{~cm}^{-1}(6.7 J)$. The mentioned features (maxima and cut-offs) of $1 \mathrm{P}$ and $2 \mathrm{P}$ excitations in the $E_{2 \mathrm{~g}}$ Raman response agree well with the theoretical prediction for the kagome QSL state ${ }^{60}$.

In more detail, the $1 \mathrm{P}$ component dominates the $E_{2 \mathrm{~g}}$ magnetic Raman continuum at low frequency. It displays the power-law behavior up to $70 \mathrm{~cm}^{-1}$, with a significantly nonmonotonic temperature dependence, as shown in Fig. 4. The low-energy 

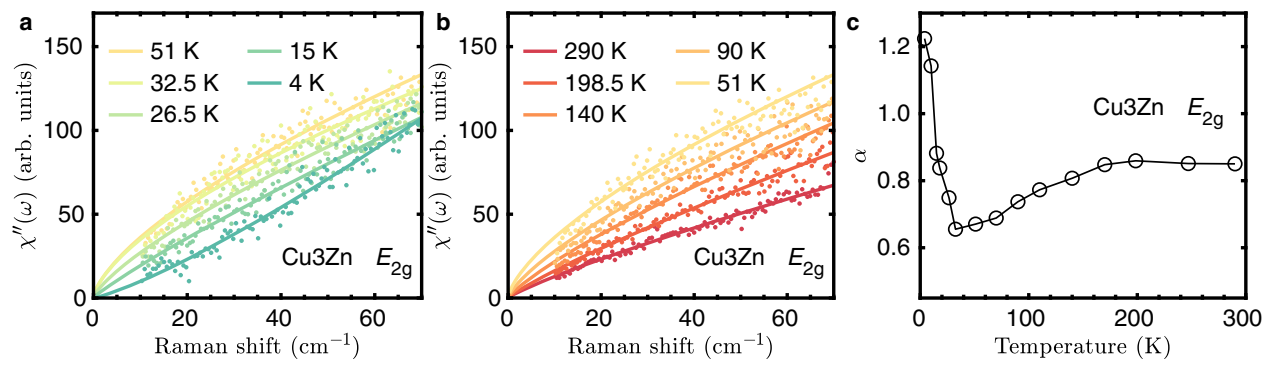

Fig. 4 Power-law behavior for $\mathbf{E}_{\mathbf{2 g}}$ magnetic Raman continua at low frequency in $\mathbf{C u}_{\mathbf{3}} \mathbf{Z n}$. $\mathbf{a}, \mathbf{b}$ are power-law fitting of $\chi_{E_{2 g}}^{\prime \prime}(\omega) \propto \omega^{\alpha}$ at low and high temperatures, respectively. c Temperature-dependent exponent $\alpha$ for the power-law fitting.

continuum evolves from a sublinear behavior $T^{\alpha}$ with $\alpha<1$ to a superlinear one $T^{\alpha}$ with $\alpha>1$ as reducing the temperature. A central question for the kagome QSL is whether a spin gap exists. Previous results on the powder samples of $\mathrm{Cu}_{3} \mathrm{Zn}$ suggest a small spin gap ${ }^{30,32}$. If such a gap exists, the power-law behavior of the $E_{2 \mathrm{~g}}$ magnetic Raman continua sets an upper bound for the spin gap of $2 \mathrm{meV}$.

The theoretical calculation for kagome Dirac spin liquid (DSL) predicts the power-law behavior for the Raman susceptibility in the $E_{2 \mathrm{~g}}$ channel at low frequency ${ }^{60}$. The $1 \mathrm{P}$ spinon excitation in DSL gives the linear density of state (DOS) $\mathcal{D}_{1 \mathrm{p}} \propto \omega$. The matrix element turns out to be exactly zero for all $1 \mathrm{P}$ excitations with $\omega$ $=0$ in the mean field Dirac Hamiltonian. As a result a Raman spectrum that scales as $\omega^{3}$ was predicted. However, the vanishing of the matrix element is somewhat accidental and depends on the assumption of a DSL in an ideal kagome Heisenberg model. Any deviation from the ideal DSL state, e.g., a small gap in the ground state $^{30,32}$, DM interactions, or other effects of perturbations ${ }^{26,67}$, changes the wave functions and may result in a constant matrix element. In that case, the Raman spectrum will be simply proportional to the DOS of the $1 \mathrm{P}$ component $\mathcal{D}_{1 \mathrm{P}}$ which is linear in $\omega$. From our fitting for $\mathrm{Cu}_{3} \mathrm{Zn}$ in Fig. 4 , we find that $\alpha=1.3$ when approaching zero temperature. The existence of a small gap in the spinon spectrum may explain the discrepancy.

Considering the interlayer $\mathrm{Cu}^{2+}$ concentration (18\%) in $\mathrm{Cu}_{3} \mathrm{Zn}$, we make a remark here about the disorder effect in the magnetic Raman scattering. The temperature-dependent $E_{2 \mathrm{~g}}$ static magnetic susceptibility $\chi_{E_{2 \mathrm{~g}}}^{\prime}(T)$ of $\mathrm{Cu}_{3} \mathrm{Zn}$ in Fig. 3 exhibit the maximal spin fluctuations at $50 \mathrm{~K}$. The non-monotonic $T$ dependence deviating from the Curie-Weiss behavior is associated with the enhancement of nearest-neighbor spin correlations at low temperatures ${ }^{67}$. However, such significant deviation from Curie-Weiss behavior is masked by the interlayer $\mathrm{Cu}^{2+}$ moments in the bulk thermodynamic measurements, e.g., heat capacity and bulk magnetization ${ }^{30}$. In contrast to a significant energy dependent magnetic Raman susceptibility $\chi_{E_{2 g}}^{\prime \prime}(\omega)$ at $4 \mathrm{~K}$ in $\mathrm{Cu}_{3} \mathrm{Zn}$, the scattered neutron signal $\chi_{\text {INS }}^{\prime \prime}(\omega)$ in Herbertsmithite is overall insensitive to energy transfer, rather flat above $1.5 \mathrm{meV}$, but increases significantly with reducing energy below $1.5 \mathrm{meV}$ due to the interlayer $\mathrm{Cu}^{2+}$ ions ${ }^{20,22}$. So Raman scattering singles out the kagome magnetic excitations and remains unmasked in the presence of the interlayer $\mathrm{Cu}^{2+}$ due to the matrix element effect as explained below. The Raman scattering measures the nearest-neighbor spin-pair $\tau_{R} \propto \mathbf{S}_{i} \cdot \mathbf{S}_{j}$ dynamics, but the spin pairs associated with the interlayer $\mathrm{Cu}^{2+}$ ions are weaker than the singlet pairs for the kagome spins. As the light polarization in our Raman measurements is in the kagome $a b$ plane, and the projected factor of the spin-pairs associated with the interlayer $\mathrm{Cu}^{2+}$ ions, $\left(\mathbf{r}_{i j} \cdot \hat{\mathbf{e}}_{\text {in }}\right)\left(\mathbf{r}_{i j} \cdot \hat{\mathbf{e}}_{\text {out }}\right)$, is small, as the related pair bond vector $\mathbf{r}_{i j}$ has the angle around $52^{\circ}$ with respect to the kagome plane. As a result, the interlayer $\mathrm{Cu}^{2+}$ ions contribute a negligible Raman matrix element and we ignore their effect in the discussions about the Raman experiments. Moreover, the inelastic neutron scattering in Herbertsmithite measures the magnetic continuum up to $3 J^{20}$, the same energy range as the $1 \mathrm{P}$ Raman component in $\mathrm{Cu}_{3} \mathrm{Zn}$. These results suggest that the magnetic Raman continuum originates from the kagome spins, and the $1 \mathrm{P}$ component has an origin of spinon excitations.

Figure 5 presents a control Raman study on the magnetic ordered kagome antiferromagnet $\mathrm{EuCu}_{3}$, which has the antiferromagnetic superexchange strength $J \simeq 7 \mathrm{meV}$. In Supplementary Note 7 , the ARPR scattering on $\mathrm{EuCu}_{3}$ confirms the threefold rotational symmetry. Above the ordering temperature $T_{\mathrm{N}}=17 \mathrm{~K}$, the magnetic Raman continuum in the $E_{\mathrm{g}}$ channel displays the extended continuum, similar to the $E_{2 \mathrm{~g}}$ magnetic continuum at $4 \mathrm{~K}$ in $\mathrm{Cu}_{3} \mathrm{Zn}$. Below $T_{\mathrm{N}}$, a sharp peak, i.e., $1 \mathrm{M}$ peak as discussed below, is observed on top of the magnetic continuum. The integrated Raman susceptibility $\chi_{E_{\mathrm{g}}}^{\prime}(T)$ monotonically increases as lowering the temperature as shown in Fig. 5b, different from non-monotonic behavior in $\chi_{E_{2 g}}^{\prime}(T)$ of $\mathrm{Cu}_{3} \mathrm{Zn}$ in Fig. 3e. The magnetic Raman susceptibility $\chi^{\prime \prime}(\omega, T)$ in $\mathrm{EuCu}_{3}$ distributes the main spectral weight among the frequency region less than $400 \mathrm{~cm}^{-1}$, and the magnon peak locates at $72 \mathrm{~cm}^{-1}$ below $17 \mathrm{~K}$, as shown in Fig. $5 \mathrm{c}$.

To directly compare the $1 \mathrm{P}$ spinon continuum in $\mathrm{Cu}_{3} \mathrm{Zn}$ and the $1 \mathrm{M}$ peak in $\mathrm{EuCu}_{3}$, we plot the $E_{\mathrm{g}}$ Raman response in $\mathrm{EuCu}_{3}$ at selected temperatures in Fig. 6. The $E_{2 g}$ Raman continuum in $\mathrm{Cu}_{3} \mathrm{Zn}$ at $4 \mathrm{~K}$ is also plotted with the proper scale for the Raman frequency. Above $T_{\mathrm{N}}=17 \mathrm{~K}, \mathrm{EuCu}_{3}$ has the substantial magnetic continuum with the profile similar to that in $\mathrm{Cu}_{3} \mathrm{Zn}$ at $4 \mathrm{~K}$. There are less pronounced low-energy continuum excitations in $\mathrm{EuCu}_{3}$ than those in $\mathrm{Cu}_{3} \mathrm{Zn}$, probably due to the large $\mathrm{DM}$ interaction which suppresses the low-energy quantum fluctuations. Below $T_{\mathrm{N}}$, a sharp magnon peak at $72 \mathrm{~cm}^{-1}$ appears in $\mathrm{EuCu}_{3}$ with the corresponding energy scale of the $1 \mathrm{P}$ continuum maximum in $\mathrm{Cu}_{3} \mathrm{Zn}$. We stress that the magnon Raman peak is direct spectroscopic evidence for the $\mathbf{q}=0120^{\circ}$ non-collinear AFM spin configurations, and invisible in the $\sqrt{3} \times \sqrt{3}$ structure of the $120^{\circ}$ AFM ("Methods").

For the AFM order state, the low-energy excitation is the spinwave magnon which can be described in the spin-wave theory ${ }^{68}$. In the local spin basis $\tilde{\mathbf{S}}_{i}$ of the AFM order, we have the Raman tensors in the XY configuration of the $E_{\mathrm{g}}$ channel for $1 \mathrm{M}$ and $2 \mathrm{M}$ components as following

$$
\begin{gathered}
\tau_{R}^{1 \mathrm{M}} \propto \sum_{R}\left(\tilde{\mathbf{S}}_{R 1}^{y}+\tilde{\mathbf{S}}_{R 2}^{y}-\tilde{\mathbf{S}}_{R 3}^{y}\right), \\
\tau_{R}^{2 \mathrm{M}} \propto \sum_{R} \tilde{\mathbf{S}}_{R 3} \odot\left(\tilde{\mathbf{S}}_{R 1}+\tilde{\mathbf{S}}_{R+\mathbf{a}_{2} 1}-\tilde{\mathbf{S}}_{R 2}-\tilde{\mathbf{S}}_{R-\mathbf{a}_{1}+\mathbf{a}_{2} 2}\right),
\end{gathered}
$$

with the $2 \mathrm{M}$ spin-pair operator $\tilde{\mathbf{S}}_{i} \odot \tilde{\mathbf{S}}_{j}=\tilde{S}_{i}^{x} \tilde{S}_{j}^{x}+\left(\tilde{S}_{i}^{y} \tilde{S}_{j}^{y}+\tilde{S}_{i}^{z} \tilde{S}_{j}^{z}\right) / 2$. For the details, please refer to the "Methods" section. Therefore, the $E_{\mathrm{g}}$ Raman scattering in the AFM order state measures $1 \mathrm{M}$ and $2 \mathrm{M}$ excitations as demonstrated in Fig. 1. Thus, the magnon 

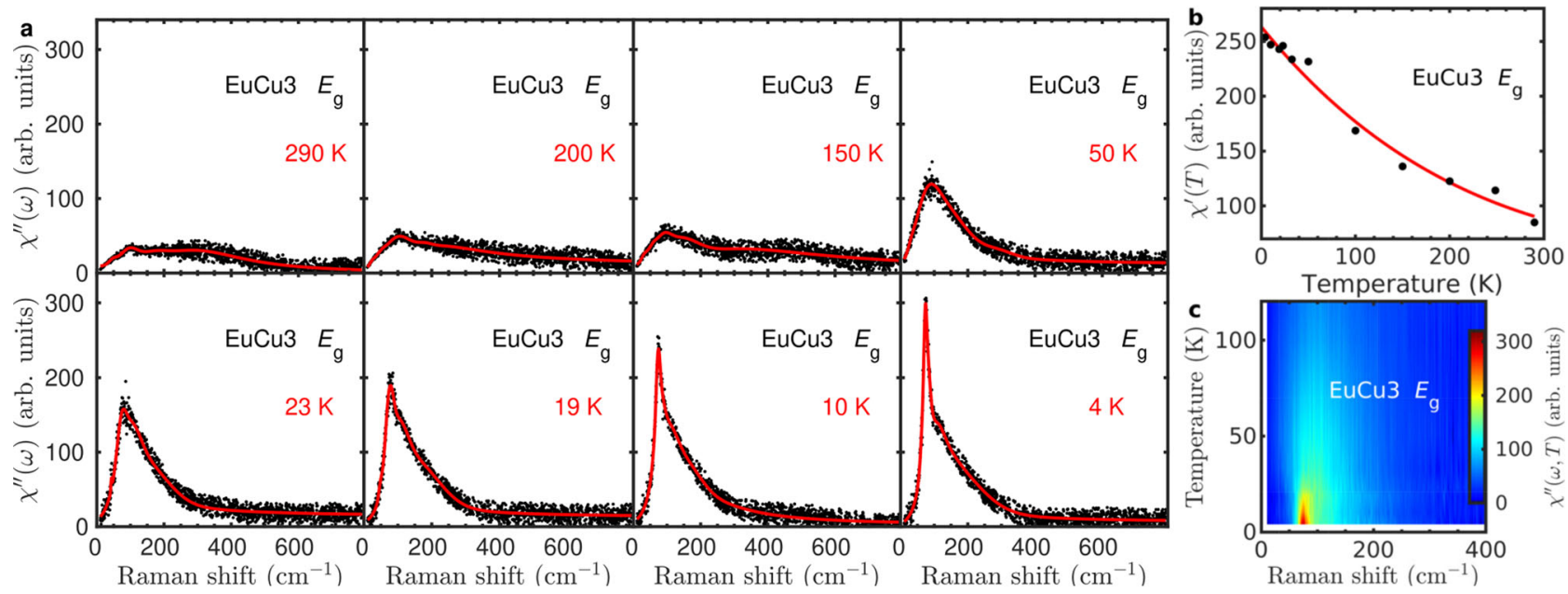

Fig. 5 Temperature dependent $\boldsymbol{E}_{\mathbf{g}}$ magnetic Raman continua in $\mathbf{E u C u}_{\mathbf{3}}$. a The $E_{\mathrm{g}}$ Raman susceptibility $\chi_{E_{\mathrm{g}}}^{\prime \prime}=\chi_{\mathrm{XY}}^{\prime \prime}$. The solid lines are guides to the eye. A sharp magnon peak appears in the $E_{\mathrm{g}}$ magnetic Raman continuum below the magnetic transition temperature $T_{\mathrm{N}}=17 \mathrm{~K}$. $\mathbf{b}$ Temperature dependence of the static Raman susceptibility in the $E_{g}$ channel $\chi_{E_{g}}^{\prime}(T)=\frac{2}{\pi} \int_{10 \mathrm{~cm}^{-1}}^{780 \mathrm{~cm}^{-1}} \frac{\chi_{E_{g}}^{\prime \prime}(\omega, T)}{\omega} d \omega$. The solid line is a guide to the eye. c Color map of $\chi_{E_{g}}^{\prime \prime}(\omega, T)$.

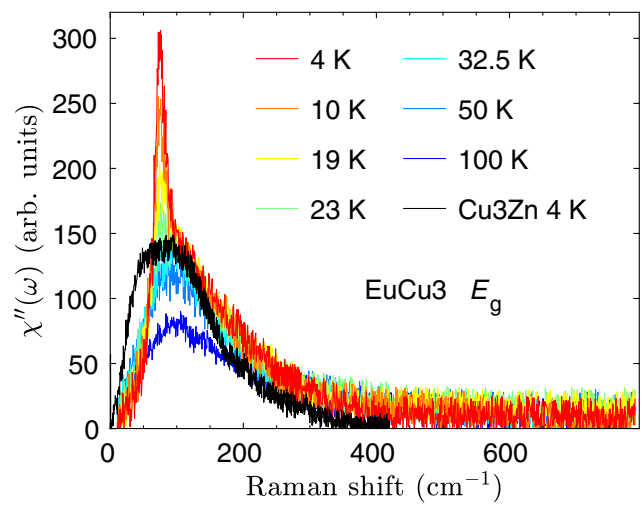

Fig. 6 Comparative Raman studies of $\mathbf{E u C u}_{3}$ and $\mathbf{C u}_{\mathbf{3}} \mathbf{Z n}$. We select the $E_{g}$ magnetic Raman continua in $\mathrm{EuCu}_{3}$ at several temperatures. For a comparison, we also plot the $E_{2 \mathrm{~g}}$ magnetic Raman continuum in $\mathrm{Cu}_{3} \mathrm{Zn}$ at 4 $\mathrm{K}$ with the Raman shift scaled by the superexchange energy ratio of 1.9.

excitation emerges from the $1 \mathrm{P}$ continuum and can be regarded as the bound state of the spinon-antispinon excitations.

\section{Discussion}

Deconfined spinons yield to the magnetic continuum, however, the Raman continuum does not necessarily imply the spin fractionalization. Only $2 \mathrm{M}$ excitation itself gives rise to a Raman continuum in the ordered antiferromagnet ${ }^{42}$. In this work, the comparative Raman study in $\mathrm{Cu}_{3} \mathrm{Zn}$ and $\mathrm{EuCu}_{3}$ resolves this uncertainty. Guided by the theoretical prediction ${ }^{60}$, the $E_{2 \mathrm{~g}}$ Raman continuum can be decomposed into $1 \mathrm{P}$ and $2 \mathrm{P}$ components of the spinon-antispinon excitations. While the $2 \mathrm{P}$ component has the maximum at $3.8 \mathrm{~J}$, resembling the $2 \mathrm{M}$ broad peak $^{42}$, the $1 \mathrm{P}$ continuum in Raman is unique for QSL. Its maximum and extended range have the same energy scale as the spin-wave magnon peak in $\mathrm{EuCu}_{3}$ and the inelastic neutron continuum cutoff (up to $3 J$ ) in the Herbertsmithite, respectively.

The $1 \mathrm{P}$ component of Raman continuum reveals fractionalized spin excitations, providing strong evidence for the kagome QSL ground state in $\mathrm{Cu}_{3} \mathrm{Zn}$. Our comparative Raman studies explicitly show the evolution from the deconfined spinon excitation in the kagome quantum spin liquid compound $\mathrm{Cu}_{3} \mathrm{Zn}$ to the conventional magnon in the kagome ordered antiferromagnet $\mathrm{EuCu}_{3}$.
On the material side, $\mathrm{Zn}$-Barlowite is an ideal structural realization of the kagome lattice. Along with Herbertsmithite, the singlecrystalline $\mathrm{Zn}$-Barlowite stands able to single out the intrinsic properties of the kagome QSL.

\section{Methods}

Sample preparation and characterization. High qualified single crystals of ZnBarlowite was grown by a hydrothermal method similar to crystal growth of herbertsmithite ${ }^{69,70}$. CuO $(0.6 \mathrm{~g}), \mathrm{ZnBr}_{2}(3 \mathrm{~g})$, and $\mathrm{NH}_{4} \mathrm{~F}(0.5 \mathrm{~g})$ and $18 \mathrm{ml}$ deionized water were sealed in a quartz tube and heated between $200^{\circ} \mathrm{C}$ and $140^{\circ} \mathrm{C}$ by a two-zone furnace. After 3 months, we obtained millimeter-sized single crystal samples. The value of $x$ in $\mathrm{Cu}_{4-x} \mathrm{Zn}_{x}(\mathrm{OH})_{6} \mathrm{FBr}$ has been determined as 0.82 by Inductively Coupled Plasma-Atomic Emission Spectroscopy (ICP-AES). The single-crystal X-ray diffraction has been carried out at room temperature by using Cu source radiation $(\lambda=1.54178 \AA)$ and solved by the Olex2.PC suite programs ${ }^{71}$. The structure and cell parameters of $\mathrm{Cu}_{4-x} \mathrm{Zn}_{x}(\mathrm{OH})_{6} \mathrm{FBr}$ are in coincidence with the previous report on polycrystalline samples ${ }^{30,32}$. For Barlowite $\left(\mathrm{Cu}_{4}(\mathrm{OH})_{6} \mathrm{FBr}\right)$, the mixture of $\mathrm{CuO}(0.6 \mathrm{~g}), \mathrm{MgBr}_{2}(1.2 \mathrm{~g})$, and $\mathrm{NH}_{4} \mathrm{~F}(0.5 \mathrm{~g})$ was transferred into Teflon-lined autoclave with $10 \mathrm{ml}$ water. The autoclave was heated up to $260^{\circ} \mathrm{C}$ and cooled to $140^{\circ} \mathrm{C}$ after 2 weeks. A similar growth condition to Barlowite was applied for the growth of $\mathrm{EuCu}_{3}(\mathrm{OH})_{6} \mathrm{Cl}_{3}$ with staring materials of $\mathrm{EuCl}_{3} \cdot 6 \mathrm{H}_{2} \mathrm{O}$ $(2 \mathrm{~g})$ and $\mathrm{CuO}(0.6 \mathrm{~g})$.

Measurement methods. Our thermodynamical measurements were carried out on the Physical Properties Measurement System (PPMS, Quantum Design) and the Magnetic Property Measurement System (MPMS3, Quantum Design).

The temperature-dependent Raman spectra are measured in a backscattering geometry using a home-modified Jobin-Yvon HR800 Raman system equipped with an electron-multiplying charged-coupled detector (CCD) and a $\times 50$ objective with long working distance and numerical aperture of 0.45 . The laser excitation wavelength is $514 \mathrm{~nm}$ from an $\mathrm{Ar}^{+}$laser. The laser-plasma lines are removed using a BragGrate bandpass filter (OptiGrate Corp.), while the Rayleigh line is suppressed using three BragGrate notch filters (BNFs) with an optical density 4 and a spectral bandwidth $\sim 5-10 \mathrm{~cm}^{-172}$. Thus, Raman signal down to $5 \mathrm{~cm}^{-1}$ can be measured ${ }^{73}$. The 1800 lines $/ \mathrm{mm}$ grating enables each CCD pixel to cover $0.6 \mathrm{~cm}^{-1}$ The samples are cooled down to $30 \mathrm{~K}$ using a Montana cryostat system under a vacuum of $0.4 \mathrm{mTorr}$ and down to $4 \mathrm{~K}$ using an attoDRY 1000 cryogenic system. All the measurements are performed with a laser power below $1 \mathrm{~mW}$ to avoid sample heating. The temperature is calibrated by the Stokes-anti-Stokes relation for the magnetic Raman continuum and phonon peaks. The intensities in two cryostat systems are matched by the Raman susceptibility. The ARPR measurements ${ }^{40}$ with light polarized in the $a b$ kagome plane of samples were performed in parallel (XX), perpendicular (XY), and X-only polarization configurations (Supplementary Note 5).

SHG measurements were performed using a homemade confocal microscope in a backscattering geometry. A fundamental wave centered at $800 \mathrm{~nm}$ was used as excitation source, which was generated from a Ti-sapphire oscillator (Chameleon Ultra II) with an $80 \mathrm{MHz}$ repetition frequency and a $150 \mathrm{fs}$ pulse width. After passing through a $\times 50$ objective, the pump beam was focused on the sample with a diameter of $2 \mu \mathrm{m}$. The scattering SHG signals at $400 \mathrm{~nm}$ were collected by the same objective and led to the entrance slit of a spectrometer equipped with a 
thermoelectrically cooled CCD. Two shortpass filters were employed to cut the fundamental wave.

Magnon Raman peak in kagome AFM ordered state. With a large DM interaction $D$, the kagome antiferromagnet in Eq. (1) devoleps a $\mathbf{q}=0$ type $120^{\circ} \mathrm{AFM}$ order at low temperature in $\mathrm{EuCu}_{3}{ }^{53-55,57-59}$. In terms of the local basis for the AFM order, we rewrite the Hamiltonian as

$$
H=J \sum_{\langle i j\rangle} \tilde{\mathbf{S}}_{i} \odot \tilde{\mathbf{S}}_{j}+D \sum_{\langle i j\rangle} \tilde{\mathbf{S}}_{i} \otimes \tilde{\mathbf{S}}_{j},
$$

with

$$
\begin{gathered}
\tilde{\mathbf{S}}_{i} \odot \tilde{\mathbf{S}}_{j}=S_{i}^{x} S_{j}^{x}+\cos \left(\theta_{i j}\right)\left(S_{i}^{y} S_{j}^{y}+S_{i}^{z} S_{j}^{z}\right)+\sin \left(\theta_{i j}\right)\left(S_{i}^{z} S_{j}^{y}-S_{i}^{y} S_{j}^{z}\right), \\
\tilde{\mathbf{S}}_{i} \otimes \tilde{\mathbf{S}}_{j}=\sin \left(\theta_{i j}\right)\left(S_{i}^{y} S_{j}^{y}+S_{i}^{z} S_{j}^{z}\right)+\cos \left(\theta_{i j}\right)\left(S_{i}^{y} S_{j}^{z}-S_{i}^{z} S_{j}^{y}\right),
\end{gathered}
$$

where $\theta_{i j}$ is an angle between two neighboring spins and $S_{i}^{x, y, z}$ below denotes the local basis of the AFM order. The effective linear spin wave Hamiltonian is given as

$$
\mathcal{H}_{\text {eff }}=J \sum_{\langle i j\rangle}\left[S_{i}^{x} S_{j}^{x}+\left(\cos \theta_{i j}+\sin \theta_{i j} D / J\right) \times\left(S_{i}^{y} S_{j}^{y}+S_{i}^{z} S_{j}^{z}\right)\right],
$$

for which the Holstein-Primakoff representation for spin operators in the local basis was applied and the energy dispersion was obtained in ref. ${ }^{68}$.

In the local spin basis, we have the Raman tensor in the XY configuration is given as

$$
\tau_{R}^{\mathrm{XY}}=\frac{\sqrt{3}}{4} \sum_{R} \tilde{\mathbf{S}}_{R 3} \odot\left(\tilde{\mathbf{S}}_{R 1}+\tilde{\mathbf{S}}_{R+\mathbf{a}_{2} 1}-\tilde{\mathbf{S}}_{R 2}-\tilde{\mathbf{S}}_{R-\mathbf{a}_{1}+\mathbf{a}_{2} 2}\right) .
$$

In the spin-pair operator $\tilde{\mathbf{S}}_{i} \odot \tilde{\mathbf{S}}_{j}$ in Eq. (8), there are two-magnon contribution in terms of $S_{i}^{x} S_{j}^{x}+\cos \left(\theta_{i j}\right)\left(S_{i}^{y} S_{j}^{y}+S_{i}^{z} S_{j}^{z}\right)$, and one- and three-magnon contributions in terms of $\sin \left(\theta_{i j}\right)\left(S_{i}^{z} S_{j}^{y}-S_{i}^{y} S_{j}^{z}\right)$. For the $\mathbf{q}=0$ spin configuration, we find that $\tau_{R}^{\mathrm{XY}}$ in Eq. (11) has the non-vanished one magnon contributions. For the $\sqrt{3} \times \sqrt{3}$ AFM state, $\tau_{R}^{\mathrm{XY}}$ has no one-magnon contribution. Therefore, the observed onemagnon peak in the $E_{\mathrm{g}}$ channel in $\mathrm{EuCu}_{3}$ provides evidence for the $\mathbf{q}=0$ spin ordering at low temperatures. In the linear spin-wave theory, we take $S^{z}$ in the local basis as a constant, $S_{i}^{z}=\left\langle S^{z}\right\rangle=1 / 2$, and the Raman tensor in XY configuration is given as

$$
\tau_{R}^{\mathrm{XY}}=\frac{3}{8} \sum_{R}\left(S_{R 1}^{y}+S_{R 2}^{y}-2 S_{R 3}^{y}\right),
$$

in terms of the local basis, directly measuring the one magnon excitation.

For $\mathrm{EuCu}_{3}$, the exchange interaction parameters are estimated as $J=7 \mathrm{meV}$, $D / J=0.3$, leading to the magnon peak position of $\Delta_{s w}=1.1 J=77 \mathrm{~cm}^{-1}$, very close to the measured value $72 \mathrm{~cm}^{-1}$ in our Raman measurement of the onemagnon peak

\section{Data availability}

All data supporting the findings of this study are available from the corresponding authors upon reasonable request.

Received: 15 December 2020; Accepted: 6 April 2021; Published online: 24 May 2021

\section{References}

1. Anderson, P. W. Resonating valence bonds: a new kind of insulator? Mater. Res. Bull. 8, 153 (1973).

2. Anderson, P. W. The resonating valence bond state in $\mathrm{La}_{2} \mathrm{CuO}_{4}$ and superconductivity. Science 235, 1196 (1987).

3. Lee, P. A. An end to the drought of quantum spin liquids. Science 321, 1306 (2008).

4. Balents, L. Spin liquids in frustrated magnets. Nature 464, 199 (2010).

5. Zhou, Y., Kanoda, K. \& Ng, T.-K. Quantum spin liquid states. Rev. Mod. Phys. 89, 025003 (2017).

6. Broholm, C. et al. Quantum spin liquids. Science 367, eaay0668 (2020).

7. Kitaev, A. Anyons in an exactly solved model and beyond. Ann. Phys. 321, 2 (2006).

8. Levin, M. \& Wen, X.-G. Detecting topological order in a ground state wave function. Phys. Rev. Lett. 96, 110405 (2006).

9. Wen, X.-G. Choreographed entanglement dances: topological states of quantum matter. Science 363, eaal3099 (2019).

10. Ran, Y., Hermele, M., Lee, P. A. \& Wen, X.-G. Projected-wave-function study of the spin-1/2 heisenberg model on the kagomé lattice. Phys. Rev. Lett. 98, 117205 (2007).
11. Hermele, M., Ran, Y., Lee, P. A. \& Wen, X.-G. Properties of an algebraic spin liquid on the kagome lattice. Phys. Rev. B 77, 224413 (2008).

12. Yan, S., Huse, D. A. \& White, S. R. Spin-liquid ground state of the $S=1 / 2$ Kagome Heisenberg antiferromagnet. Science 332, 1173 (2011).

13. Jiang, H.-C., Wang, Z. \& Balents, L. Identifying topological order by entanglement entropy. Nat. Phys 8, 902 (2012).

14. Zaletel, M. P. \& Vishwanath, A. Constraints on topological order in mott insulators. Phys. Rev. Lett. 114, 077201 (2015).

15. Mei, J.-W., Chen, J.-Y., He, H. \& Wen, X.-G. Gapped spin liquid with $\mathbb{Z}_{2}$ topological order for the kagome Heisenberg model. Phys. Rev. B 95, 235107 (2017).

16. Norman, M. R. Colloquium: Herbertsmithite and the search for the quantum spin liquid. Rev. Mod. Phys. 88, 041002 (2016).

17. Shores, M. P., Nytko, E. A., Bartlett, B. M. \& Nocera, D. G. A structurally perfect $S=1 / 2$ kagome antiferromagnet. J. Am. Chem. Soc. 127, 13462 (2005).

18. Mendels, P. et al. Quantum magnetism in the paratacamite family: towards an ideal kagomé lattice. Phys. Rev. Lett. 98, 077204 (2007).

19. Wulferding, D. et al. Interplay of thermal and quantum spin fluctuations in the kagome lattice compound herbertsmithite. Phys. Rev. B 82, 144412 (2010).

20. Han, T.-H. et al. Fractionalized excitations in the spin-liquid state of a kagome-lattice antiferromagnet. Nature 492, 406 (2012).

21. Fu, M., Imai, T., Han, T.-H. \& Lee, Y. S. Evidence for a gapped spin-liquid ground state in a kagome heisenberg antiferromagnet. Science 350, 655 (2015).

22. Han, T.-H. et al. Correlated impurities and intrinsic spin-liquid physics in the kagome material herbertsmithite. Phys. Rev. B 94, 060409 (2016).

23. Khuntia, P. et al. Gapless ground state in the archetypal quantum kagome antiferromagnet $\mathrm{ZnCu}_{3}(\mathrm{OH})_{6} \mathrm{Cl}_{2}$. Nat. Phys. 16, 469 (2020).

24. Zorko, A. et al. Symmetry reduction in the quantum kagome antiferromagnet herbertsmithite. Phys. Rev. Lett. 118, 017202 (2017).

25. Laurita, N. J. et al. Evidence for a parity broken monoclinic ground state in the $\mathrm{S}=1 / 2$ kagomé antiferromagnet herbertsmithite. Preprint at https://arxiv.org/ abs/1910.13606 (2019).

26. Norman, M. R., Laurita, N. J. \& Hsieh, D. Valence bond phases of herbertsmithite and related copper kagome materials. Phys. Rev. Res. 2, 013055 (2020).

27. Li, Y. et al. Lattice dynamics in the spin- $\frac{1}{2}$ frustrated kagome compound herbertsmithite. Phys. Rev. B 101, 161115 (2020).

28. Han, T.-H., Singleton, J. \& Schlueter, J. A. Barlowite: a spin-1/2 antiferromagnet with a geometrically perfect kagome motif. Phys. Rev. Lett. 113, 227203 (2014).

29. Liu, Z., Zou, X., Mei, J.-W. \& Liu, F. Selectively doping barlowite for quantum spin liquid: a first-principles study. Phys. Rev. B 92, 220102 (2015).

30. Feng, Z. et al. Gapped spin-1/2 spinon excitations in a new kagome quantum spin liquid compound $\mathrm{Cu}_{3} \mathrm{Zn}(\mathrm{OH})_{6}$ FBr. Chinese Phys. Lett. 34, 077502 (2017).

31. Feng, Z. et al. Effect of $\mathrm{Zn}$ doping on the antiferromagnetism in kagome $\mathrm{Cu}_{4-x}$ $\mathrm{Zn}_{x}(\mathrm{OH})_{6}$ FBr. Phys. Rev. B 98, 155127 (2018).

32. Wei, Y. et al. Evidence for a $Z_{2}$ topological ordered quantum spin liquid in a kagome-lattice antiferromagnet. Preprint at https://arxiv.org/abs/1710.02991 (2017).

33. Henderson, A. et al. Order-disorder transition in the $S=\frac{1}{2}$ kagome antiferromagnets claringbullite and barlowite. Chem. Commun. 55, 11587 (2019).

34. Pasco, C. et al. Single-crystal growth of $\mathrm{Cu}_{4}(\mathrm{OH})_{6} \mathrm{BrF}$ and universal behavior in quantum spin liquid candidates synthetic barlowite and herbertsmithite. Phys. Rev. Mater. 2, 044406 (2018).

35. Smaha, R. W., He, W., Sheckelton, J. P., Wen, J. \& Lee, Y. S. Synthesisdependent properties of barlowite and Zn-substituted barlowite. J. Solid State Chem. 268, 123 (2018).

36. Smaha, R. W. et al. Materializing rival ground states in the barlowite family of kagome magnets: quantum spin liquid, spin ordered, and valence bond crystal states. npj Quantum Mater. 5, 1 (2020).

37. Tustain, K. et al. From magnetic order to quantum disorder in the $\mathrm{Zn}$ barlowite series of $S=\frac{1}{2}$ kagomé antiferromagnets. npj Quantum Mater. 5, 74 (2020).

38. Wei, Y. et al. Magnetic phase diagram of $\mathrm{Cu}_{4-x} \mathrm{Zn}_{x}(\mathrm{OH})_{6} \mathrm{FBr}$ studied by neutron-diffraction and $\mu \mathrm{SR}$ techniques. Chinese Phys. Lett. 37, 107503 (2020)

39. Cépas, O., Haerter, J. O. \& Lhuillier, C. Detection of weak emergent broken symmetries of the kagome antiferromagnet by Raman spectroscopy. Phys. Rev. B 77, 172406 (2008)

40. Liu, X.-L., Zhang, X., Lin, M.-L. \& Tan, P.-H. Different angle-resolved polarization configurations of raman spectroscopy: A case on the basal and edge plane of two-dimensional materials. Chin. Phys. B 26, 067802 (2017).

41. Lemmens, P., Güntherodt, G. \& Gros, C. Magnetic light scattering in lowdimensional quantum spin systems. Physics Reports 375, 1 (2003).

42. Devereaux, T. P. \& Hackl, R. Inelastic light scattering from correlated electrons. Rev. Mod. Phys. 79, 175 (2007). 
43. Wulferding, D., Choi, Y., Lee, W. \& Choi, K.-Y. Raman spectroscopic diagnostic of quantum spin liquids. J. Phys. Condens. Mat. 32, 043001 (2019).

44. Sandilands, L. J., Tian, Y., Plumb, K. W., Kim, Y.-J. \& Burch, K. S. Scattering continuum and possible fractionalized excitations in $\alpha-\mathrm{RuCl}_{3}$. Phys. Rev. Lett. 114, 147201 (2015).

45. Glamazda, A., Lemmens, P., Do, S. H., Choi, Y. S. \& Choi, K. Y. Raman spectroscopic signature of fractionalized excitations in the harmonichoneycomb iridates $\beta$ - and $\gamma-\mathrm{Li}_{2} \mathrm{IrO}_{3}$. Nat. Commun. 7, 12286 (2016).

46. Glamazda, A., Lemmens, P., Do, S. H., Kwon, Y. S. \& Choi, K. Y. Relation between Kitaev magnetism and structure in $\alpha-\mathrm{RuCl}_{3}$. Phys. Rev. B 95, 174429 (2017).

47. Li, G. et al. Raman spectroscopy evidence for dimerization and Mott collapse in $\alpha-\mathrm{RuCl}_{3}$ under pressures. Phys. Rev. Mater. 3, 023601 (2019).

48. Pei, S. et al. Magnetic Raman continuum in single-crystalline $\mathrm{H}_{3} \mathrm{LiIr}_{2} \mathrm{O}_{6}$. Phys. Rev. B 101, 201101 (2020).

49. Wulferding, D. et al. Magnon bound states versus anyonic Majorana excitations in the Kitaev honeycomb magnet $\alpha-\mathrm{RuCl}_{3}$. Nat. Commun. 11, 1603 (2020).

50. Wang, Y. et al. The range of non-Kitaev terms and fractional particles in $\alpha$ - $\mathrm{RuCl}_{3}$. npj Quantum Mater. 5, 14 (2020).

51. Sun, W., Huang, Y.-X., Nokhrin, S., Pan, Y. \& Mi, J.-X. Perfect Kagomé lattices in $\mathrm{YCu}_{3}(\mathrm{OH})_{6} \mathrm{Cl}_{3}$ : a new candidate for the quantum spin liquid state. J. Mater. Chem. 4, 8772 (2016).

52. Sun, W., Huang, Y. X., Pan, Y. \& Mi, J. X. Strong spin frustration and negative magnetization in $\mathrm{LnCu}_{3}(\mathrm{OH})_{6} \mathrm{Cl}_{3}(\mathrm{Ln}=\mathrm{Nd}$ and $\mathrm{Sm})$ with triangular lattices: the effects of lanthanides. Dalton Trans. 46, 9535 (2017).

53. Puphal, P., Zoch, K. M., Désor, J., Bolte, M. \& Krellner, C. Kagome quantum spin systems in the atacamite family. Phys. Rev. Mater. 2, 063402 (2018).

54. Zorko, A. et al. Negative-vector-chirality $120^{\circ}$ spin structure in the defect- and distortion-free quantum kagome antiferromagnet $\mathrm{YCu}_{3}(\mathrm{OH})_{6} \mathrm{Cl}_{3}$. Phys. Rev. B 100, 144420 (2019).

55. Arh, T. et al. Origin of magnetic ordering in a structurally perfect quantum kagome antiferromagnet. Phys. Rev. Lett. 125, 027203 (2020).

56. Zorko, A. et al. Dzyaloshinsky-Moriya anisotropy in the spin-1/2 kagome compound $\mathrm{ZnCu}_{3}(\mathrm{OH})_{6} \mathrm{Cl}_{2}$. Phys. Rev. Lett. 101, 026405 (2008).

57. Cépas, O., Fong, C. M., Leung, P. W. \& Lhuillier, C. Quantum phase transition induced by Dzyaloshinskii-Moriya interactions in the kagome antiferromagnet. Phys. Rev. B 78, 140405 (2008).

58. Rousochatzakis, I., Manmana, S. R., Läuchli, A. M., Normand, B. \& Mila, F. Dzyaloshinskii-Moriya anisotropy and nonmagnetic impurities in the $s=\frac{1}{2}$ kagome system $\mathrm{ZnCu}_{3}(\mathrm{OH})_{6} \mathrm{Cl}_{2}$. Phys. Rev. B 79, 214415 (2009).

59. Zhu, W., Gong, S.-S. \& Sheng, D. N. Identifying spinon excitations from dynamic structure factor of spin-1/2 Heisenberg antiferromagnet on the Kagome lattice. Proc. Natl. Acad. Sci. U.S. 116, 5437 (2019).

60. Ko, W.-H., Liu, Z.-X., Ng, T.-K. \& Lee, P. A. Raman signature of the U(1) Dirac spin-liquid state in the spin- $\frac{1}{2}$ kagome system. Phys. Rev. B 81, 024414 (2010).

61. Freedman, D. E. et al. Site specific X-ray anomalous dispersion of the geometrically frustrated KAGomé magnet, herbertsmithite, $\mathrm{ZnCu}_{3}(\mathrm{OH})_{6} \mathrm{Cl}_{2}$. J. Am. Chem. Soc. 132, 16185 (2010).

62. Helton, J. S. et al. Spin dynamics of the spin-1/2 kagome lattice antiferromagnet $\mathrm{ZnCu}_{3}(\mathrm{OH})_{6} \mathrm{Cl}_{2}$. Phys. Rev. Lett. 98, 107204 (2007).

63. Halley, J. W. Light scattering as a probe of dynamical critical properties of antiferromagnets. Phys. Rev. Lett. 41, 1605 (1978).

64. Reiter, G. F. Light scattering from energy fluctuations in magnetic insulators. Phys. Rev. B 13, 169 (1976).

65. Fleury, P. A. \& Loudon, R. Scattering of light by one- and two-Magnon excitations. Phys. Rev. 166, 514 (1968).

66. Shastry, B. S. \& Shraiman, B. I. Theory of Raman scattering in Mott-Hubbard systems. Phys. Rev. Lett. 65, 1068 (1990).

67. Bernu, B., Pierre, L., Essafi, K. \& Messio, L. Effect of perturbations on the kagome $S=\frac{1}{2}$ antiferromagnet at all temperatures. Phys. Rev. B 101, 140403 (2020).

68. Chernyshev, A. L. \& Zhitomirsky, M. E. Quantum selection of order in an XXZ antiferromagnet on a kagome lattice. Phys. Rev. Lett. 113, 237202 (2014).

69. Chu, S., Müller, P., Nocera, D. G. \& Lee, Y. S. Hydrothermal growth of single crystals of the quantum magnets: Clinoatacamite, paratacamite, and herbertsmithite. Appl. Phys. Lett. 98, 092508 (2011).
70. Velázquez, M. et al. Aqueous solution growth at $200{ }^{\circ} \mathrm{C}$ and characterizations of pure, ${ }^{17} \mathrm{O}$-or D-based herbertsmithite $\mathrm{Zn}_{x} \mathrm{Cu}_{4-x}(\mathrm{OH})_{6} \mathrm{Cl}_{2}$ single crystals. J. Cryst. Growth 531, 125372 (2020).

71. Dolomanov, O. V., Bourhis, L. J., Gildea, R. J., Howard, J. A. \& Puschmann, H. Olex2: a complete structure solution, refinement and analysis program. J. Appl. Crystallogr. 42, 339 (2009).

72. Tan, P. H. et al. The shear mode of multilayer graphene. Nat. Mater. 11, 294 (2012).

73. Lin, M.-L. et al. Cross-dimensional electron-phonon coupling in van der Waals heterostructures. Nat. Commun. 10, 2419 (2019).

\section{Acknowledgements}

This work was supported by the National Key Research and Development Program of China (2016YFA0301204), the program for Guangdong Introducing Innovative and Entrepreneurial Teams (No. 2017ZT07C062), by Shenzhen Key Laboratory of Advanced Quantum Functional Materials and Devices (No. ZDSYS20190902092905285), Guangdong Natural Science Foundation (No. 2020B1515120100) and by National Natural Science Foundation of China (Grant Nos. 11774143, 12004377 and 11874350), the CAS Key Research Program of Frontier Sciences (ZDBS-LY-SLH004) and China Postdoctoral Science Foundation (2019TQ0317 and 2020M682780). P.A. Lee acknowledges support by the US Department of Energy under grant number DE-FG02-03ER46076.

\section{Author contributions}

J.W.M. conceived the project. P.H.T. conceived the experimental work of Raman spectroscopy. Y.F., L.W., L.H., W.J., and Z.H. synthesized single crystals of samples. M.L. and P.H.T. designed the Raman experiments. M.L., J.Z., and P.H.T. performed Raman measurements. Q.L. and J.D. performed the SHG measurements. Y.F., L.W., L.H., and C.L. performed and analyzed magnetic susceptibility and heat capacity measurements. H.Z., X.S., and J.W.M. performed first-principles calculations. J.W.M., Y.F., M.L., and P.H.T. analyzed the Raman data. P.A.L., J.W.M., and F.Y. worked on the theory. P.A.L., J.W.M., F.Y., P.H.T., and M.L.L. wrote the manuscript with contributions and comments from all authors.

\section{Competing interests}

The authors declare no competing interests.

\section{Additional information}

Supplementary information The online version contains supplementary material available at https://doi.org/10.1038/s41467-021-23381-9.

Correspondence and requests for materials should be addressed to P.-H.T. or J.-W.M.

Peer review information Nature Communications thanks the anonymous reviewers for their contribution to the peer review of this work. Peer reviewer reports are available.

Reprints and permission information is available at http://www.nature.com/reprints

Publisher's note Springer Nature remains neutral with regard to jurisdictional claims in published maps and institutional affiliations.

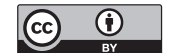

Open Access This article is licensed under a Creative Commons Attribution 4.0 International License, which permits use, sharing, adaptation, distribution and reproduction in any medium or format, as long as you give appropriate credit to the original author(s) and the source, provide a link to the Creative Commons license, and indicate if changes were made. The images or other third party material in this article are included in the article's Creative Commons license, unless indicated otherwise in a credit line to the material. If material is not included in the article's Creative Commons license and your intended use is not permitted by statutory regulation or exceeds the permitted use, you will need to obtain permission directly from the copyright holder. To view a copy of this license, visit http://creativecommons.org/ licenses/by/4.0/.

(c) The Author(s) 2021 